\title{
Near-Comprehensive Resequencing of Cancer-Associated Genes in Surgically Resected Metastatic Liver Tumors of Gastric Cancer
}

\author{
Naoki Ikari, ${ }^{* \dagger}$ Akiko Serizawa, ${ }^{*}$ Shohei Mitani, ${ }^{\ddagger}$ Masakazu Yamamoto, ${ }^{*}$ and Toru Furukawa ${ }^{\dagger \S \pi}$
}

From the Department of Surgery, ${ }^{*}$ Institute of Gastroenterology, and the Institute for Integrated Medical Sciences, ${ }^{\dagger}$ Tokyo Women's Medical University, Tokyo; the Department of Physiology, ${ }^{\ddagger}$ Tokyo Women’s Medical University School of Medicine, Tokyo; the Department of Surgical Pathology, ${ }^{\S}$ Tokyo Women's Medical University Hospital, Tokyo; and the Department of Histopathology, ${ }^{\circledR}$ Tohoku University Graduate School of Medicine, Sendai, Japan

\author{
Accepted for publication \\ December 6, 2018. \\ Address correspondence to \\ Toru Furukawa, M.D., Ph.D., \\ Department of Histopathology, \\ Tohoku University Graduate \\ School of Medicine, 2-1 Seir- \\ yomachi, Aoba-ku, Sendai 980- \\ 8575, Japan. E-mail: toru. \\ furukawa@med.tohoku.ac.jp.
}

\begin{abstract}
Liver metastasis is a major cause of death in patients with gastric cancer. The molecular alterations in clinically resected liver metastases of gastric cancer were evaluated to identify candidate biomarkers and therapeutic targets. Seventy-four patients, including 37 with liver metastasis who underwent gastrectomy and hepatectomy for gastric cancer and 37 without liver metastasis who underwent gastrectomy for gastric cancer, were studied. Next-generation resequencing was performed for 412 cancerassociated genes in metastatic and/or primary tumors from 30 patients and somatic mutations in TP53, LRP1B, PIK3CA, ADAMTS20, PAX7, FN1, FOX03, WRN, PTEN, ETV4, and RNF213 were found in metastatic tumors. TP53 mutations were studied by Sanger sequencing in the remaining patients; the number of patients with TP53 mutations in metastatic tumors was significantly higher among those with liver metastasis $(86.5 \%, 32 / 37)$ versus those without liver metastasis $(40.5 \% ; 15 / 37 ; P<0.0001)$. TP53 mutations in metastatic liver tumors and corresponding primary tumors were identical in $96.9 \%$ (31) 32 ), including some patients with heterogeneous primary tumor components. Immunohistochemical analyses showed aberrant p53 expression in tumors with TP53 mutations. In silico functional evaluations indicated functional loss of missense-mutated TP53. Thus, the p53 pathway may facilitate the development of biomarkers and therapeutic approaches to treat gastric cancer metastases to the liver. (Am J Pathol 2019, 189: 784-796; https://doi.org/10.1016/j.ajpath.2018.12.015)
\end{abstract}

Gastric cancer is the fifth most frequent and the third most lethal cancer in the world (Global Cancer Observatory, http://globocan.iarc.fr, last accessed June 2, 2018). Although regionally localized gastric cancers may be treated with endoscopic resection or surgery with lymphadenectomy, those with liver metastases are difficult to treat and, hence, fatal. ${ }^{1-6}$ To overcome this intractable situation, it is fundamental to know the molecular basis of liver metastasis of gastric cancer, necessitating the direct investigation of the molecular alterations in clinical samples of metastatic tumors. However, such clinical samples of liver metastases from gastric cancer are rarely obtained by surgical resection because the commonly recommended treatments for metastatic disease include chemotherapy, radiation, palliative surgery, and/or best supportive care. In addition, the retrospective clinical outcomes of limited surgical cases (median: 25, range, 15 to 64) have been reported as $0 \%$ to $37 \%$ in the 5 -year survival rate. ${ }^{7-9}$

Under these circumstances, the molecular basis of liver metastases from gastric cancer has remained largely unknown. There have been two case reports showing comprehensive analysis of the molecular alterations in distant metastases from gastric cancer, wherein the distant metastases was observed, not in the liver, but in the peritoneum. $^{10,11}$

We have recently demonstrated that histologic uniformity of primary tumor may serve as a favorable prognostic factor for patients undergoing resection of liver metastasis from

Partially supported by Japan Society for the Promotion of Science Grantin-Aid for Scientific Research JP16K10518 (A.S.).

Disclosures: None declared. 
gastric cancer, suggestive of the subgroup suitable for surgical resection. ${ }^{12}$ Here, the authors expanded their research on liver metastasis of gastric cancer by analyzing 74 patients, including 37 patients with liver metastasis that underwent gastrectomy and hepatectomy for gastric cancer and 37 patients without liver metastasis that underwent gastrectomy for gastric cancer, for molecular alterations in the metastatic and/or primary tumors using next-generation resequencing and additional multimodal analyses to identify the candidate molecular biomarkers and therapeutic targets for liver metastasis of gastric cancer.

\section{Materials and Methods}

\section{Patients}

This study was approved by the ethics committee of Tokyo Women's Medical University on November 4, 2014 (protocol \#272). Seventy-four patients with gastric cancer operated on at the Tokyo Women's Medical University Hospital were retrospectively studied. Of these, 37 had consecutive gastric cancer and liver metastasis and had undergone gastric and hepatic resections either synchronously or metachronously between 1990 and 2017 (Supplemental Figure S1). The remaining 37 were patients with gastric cancer that had undergone gastrectomy between 2005 and 2010, and had experienced no liver metastasis for over 5 years; these patients were retrospectively selected to have primary tumors with the same $\mathrm{T}$ stage as the primary tumors in the liver metastasis patients (Supplemental Figure $\mathrm{S} 1$ ). The $\mathrm{T}$ stage was determined according to the eighth edition of the AJCC Cancer Staging Manual. ${ }^{13}$

\section{Tissue Dissection and DNA Preparation}

Formalin-fixed and paraffin-embedded (FFPE) tissue samples of primary gastric tumors, metastatic liver tumors, and normal portions of the stomach (submucosa or deeper areas) were used for genetic analysis. Areas of adenocarcinoma accessed by microscopic examination were manually dissected from unstained, $10-\mu \mathrm{m}-$ thick tissue sections. Tumors with several different histologic components with clear borders were separately dissected to investigate clonal variations. DNA was extracted using the QIAamp DNA FFPE Tissue Kit (Qiagen, Hilden, Germany) according to the manufacturer's instructions.

\section{Next-Generation Resequencing in a Discovery Set}

Two panels of cancer-associated genes targeted for nextgeneration resequencing, namely, the Ion AmpliSeq Comprehensive Cancer Panel (Thermo Fisher Scientific, Carlsbad, CA) that covered all coding exons and splice sites of 409 cancer-associated genes and the Ion AmpliSeq Custom DNA Panel (Thermo Fisher Scientific) designed to cover all coding exons and splice sites of RHOA and its regulators $A K A P 13$ and $D L C$, were used as previously described. ${ }^{14}$ Library constructions and sequencing was performed as previously described. ${ }^{14}$

\section{Variant Calling and Annotations}

Data analyses were performed in the Ion Torrent Suite software version 5.0.3 (Thermo Fisher Scientific). After base calling, sequencing reads were aligned against the reference human genome (hg19) with the TMAP algorithm within the Torrent Suite. Variants fulfilling the filtering criteria shown in Supplemental Table S1 were counted; a $10 \%$ allele frequency threshold was set according to the sensitivity of the traditional PCR method applied to the remaining validation set. ${ }^{15}$ For single nucleotide polymorphism analysis, nonsynonymous nucleotide substitutions detected only in the tumor tissues were counted; however, single nucleotide polymorphisms at a frequency of $>1 \%$ in the 1000G, ExAC, or ESP6500si database were dismissed, because these single nucleotide polymorphisms were considered to be false-positive somatic mutations. Functional effects of mutations and their clinical relevance were annotated using ClinVar, SIFT, Polyphen2, and OncoKB. ${ }^{16-19}$ The variants counted as described above were validated by means of the Sanger sequencing according to the previously described method. ${ }^{20}$ The primers used for Sanger sequencing are listed in Table 1.

\section{Mutational Analysis of the Validation Set}

As a validation set, the coding regions and splice sites of TP53 were examined with the Sanger sequencing method using the primers listed in Table $1 .^{20}$

\section{Expression Analysis for $\mathrm{p} 53$}

Expression of p53 was examined by immunohistochemical analysis. FFPE tissue sections were deparaffinized with xylene, hydrated with serially diluted ethanol, and then heat-pretreated in $10 \mathrm{mmol} / \mathrm{L}$ sodium citrate buffer. The tissues were incubated with the mouse monoclonal antip53 antibody (clone DO-7; Dako, Glostrup, Denmark) diluted at 1:500 concentration in 10\% normal rabbit serum solution (Nichirei Biosciences, Tokyo, Japan). Serial sections incubated with $10 \%$ normal serum solution without the primary antibody were used as the negative control. The biotin-conjugated rabbit anti-mouse IgG + IgA + IgM (Nichirei Biosciences) was used as a secondary antibody. After incubation with peroxidase-conjugated streptavidin (Nichirei Biosciences), 3,3'-diaminobenzidine chromogen solution (Dako) was used for visualization. Nuclei were counterstained with hematoxylin. p53 expression in immunohistochemical analysis was evaluated as overexpression, complete absence of expression or weak expression, cytoplasmic expression, or scattered expression. 
Table 1 Primer Sequences Used in Sanger Sequencing

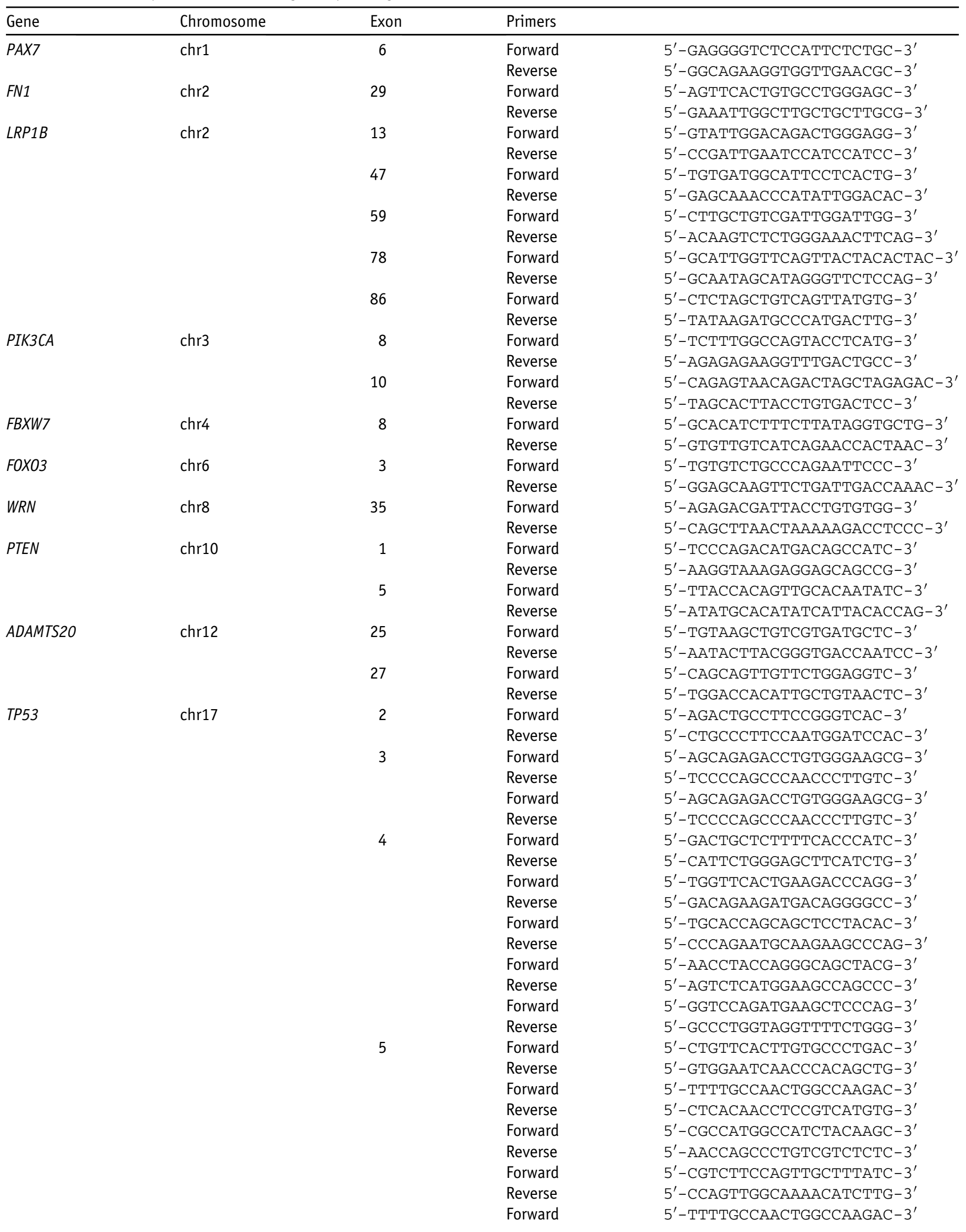

(table continues) 
Table 1 (continued)

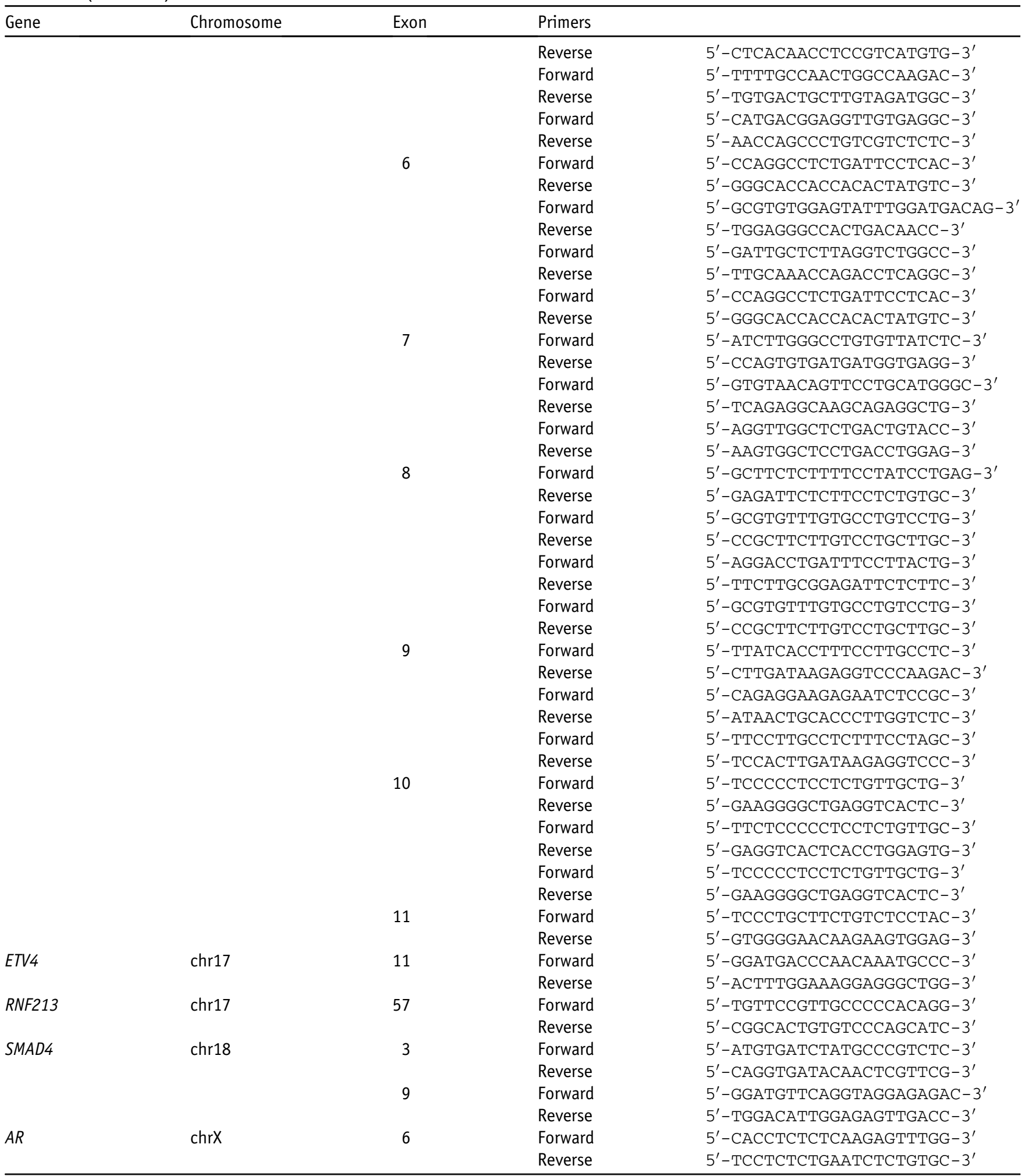

\section{In Silico Functional Analysis of Mutant p53}

The International Agency for Research on Cancer (IARC) TP53 Database (http://p53.iarc.fr, last accessed June 4, 2018) was used for the in silico analysis of mutant $\mathrm{p} 53 .^{21}$

\section{Definitions}

The metastatic group comprised patients with liver metastasis, whereas the nonmetastatic group comprised patients without liver metastasis regardless of other metastases such 
as lymph node metastasis. Tumor status was determined according to the 8th edition of the AJCC Cancer Staging Manual. ${ }^{13}$ Histologic types were evaluated according to Lauren's classification, that is, the intestinal type and the diffuse type. ${ }^{22}$ A primary tumor with a mixture of discrete histologic components of more than $30 \%$ of the total was regarded as the mixed histologic type. In-frame deletions were categorized into nontruncating mutations.

\section{Statistical Analyses}

Continuous data were expressed as the median with or without maximum and minimum values. The relation between two variables was analyzed by Fisher's exact test or the logistic regression analysis according to the scale. Spearman's rank correlation coefficient was used to determine the strength of the relationship between TP53 mutations in liver metastasis and clinicopathologic features. A value of $P<0.05$ was considered statistically significant. All the statistical analyses were performed using JMP Pro software version 12 (SAS Institute, Cary, NC).

\section{Results}

\section{Clinicopathologic Features of 74 Patients}

Seventy-four patients were analyzed. The patients were classified into two groups: a metastatic group with 37 patients with liver metastasis who underwent gastrectomy and synchronous or metachronous hepatectomy for gastric cancer; and a nonmetastatic group with 37 patients who underwent only gastrectomy for gastric cancer in the equivalent $\mathrm{T}$ stage to the primary tumors in those from the metastatic group (Supplemental Figure S1 and Supplemental Table S2). The comparison of the clinicopathologic features revealed that the patients in the metastatic group showed higher $\mathrm{N}$ stage, more frequent venous invasion, and more frequent intestinal-type primary tumors $(P=0.0015, P<0.0001$, and $P=0.0022$, respectively $)$ than those in the nonmetastatic group (Table 2). Serum carcinoembryonic antigen (CEA), carbohydrate antigen 199 (CA19-9), and alpha fetoprotein (AFP) levels were more frequently elevated in the patients from the metastatic group than in those from the nonmetastatic group $(P=0.0275$, $P=0.0317$, and $P=0.0166$, respectively) (Table 2).

\section{Mutated Genes Identified in the Discovery Set of 30 Patients}

To explore the candidate genes responsible for liver metastasis of gastric cancer, a discovery set comprising 30 patients, including 15 patients from each of the metastatic and nonmetastatic groups, was studied. Tumors of these patients were assessed with next-generation resequencing for protein-coding exons and splice sites of 412 cancerassociated genes, including 409 genes from the Ion
AmpliSeq Comprehensive Cancer Panel and 3 genes (RHOA, AKAP13, and DLC) from the Ion AmpliSeq Custom DNA Panel. The median coverage values for both gene sets were 399-fold (range, 336- to 561-fold) and 4706fold (range, 1533- to 10,496-fold), respectively.

Somatic mutations were identified in metastatic liver tumors in TP53, LRP1B, PIK3CA, ADAMTS20, PAX7, FN1, FOXO3, WRN, PTEN, ETV4, and RNF213. Of these somatic mutations, TP53 mutations were more frequently found in the metastatic group than in the nonmetastatic group. In particular, various TP53 mutations were found in 13 of 15 patients in the metastatic group and 6 of 15 patients in the nonmetastatic group $(P=0.0209)$ (Table 3$)$. Among the other mutations found in the discovery set, the following mutations appeared to be selected in the course of metastasis from heterogeneous clones within primary tumors showing mixed histology: LRPIB (splicing, c.7642+1G>T), PIK3CA (p.E545K), PAX7 (p.R269C), FN1 (p.T1504fs), FOXO3 (p.D514N), WRN (p.T1425M), ETV4 (p.L371V), and RNF213 (p.F4615L) (Table 3). PIK3CA (p.P466L) and PTEN (p.Q17*) were detected in metastatic liver tumor, but not in the corresponding primary tumor (Table 3). The mutations found only in the patients from the nonmetastatic group included $L R P 1 B$ (p.E666D), LRP1B (p.L3116P), PIK3CA (p.E542K), PIK3CA (p.E542G), SMAD4 (p.A118fs), SMAD4 (p.R361C), PTEN (p.W111*), and AR (p.F814V). All these mutations were validated by Sanger sequencing (Supplemental Figures S2 and S3).

\section{TP53 Mutations in Tumors of 74 Patients}

As TP53 mutations were significantly more frequent in the metastatic group than in the nonmetastatic group among the 30 patients from the discovery set, tumor samples from the rest of 44 patients were investigated for mutations in all coding exons and splice sites of TP53 by Sanger sequencing as a validation set. In the validation set, $86.4 \%(19 / 22)$ and $40.9 \%(9 / 22)$ of patients from the metastatic group and nonmetastatic groups, respectively, harbored TP53 mutations in tumors. In total, $86.5 \%(32 / 37)$ of patients from the metastatic group harbored TP53 mutations in the metastatic liver tumors (Figure 1A and Supplemental Table S3). The frequency of TP53 mutations was significantly higher in the patients from the metastatic group than in those from the nonmetastatic group, of which 40.5\% (15/37) harbored TP53 mutations in their primary tumors $(P<0.0001)$. According to The Cancer Genome Atlas (TCGA) data, 47.4\% (136/287) of patients with gastric cancer harbored TP53 mutations in their primary tumors $^{23-25}$; this number was significantly lower than that reported in patients from the metastatic group of the study cohort $(P<0.0001)$ (Figure $1 \mathrm{~A})$. Furthermore, 96.9\% (31/32) of the patients harboring TP53 mutations in metastatic liver tumors had the same TP53 mutations 
Table 2 Clinicopathologic Features of 74 Patients

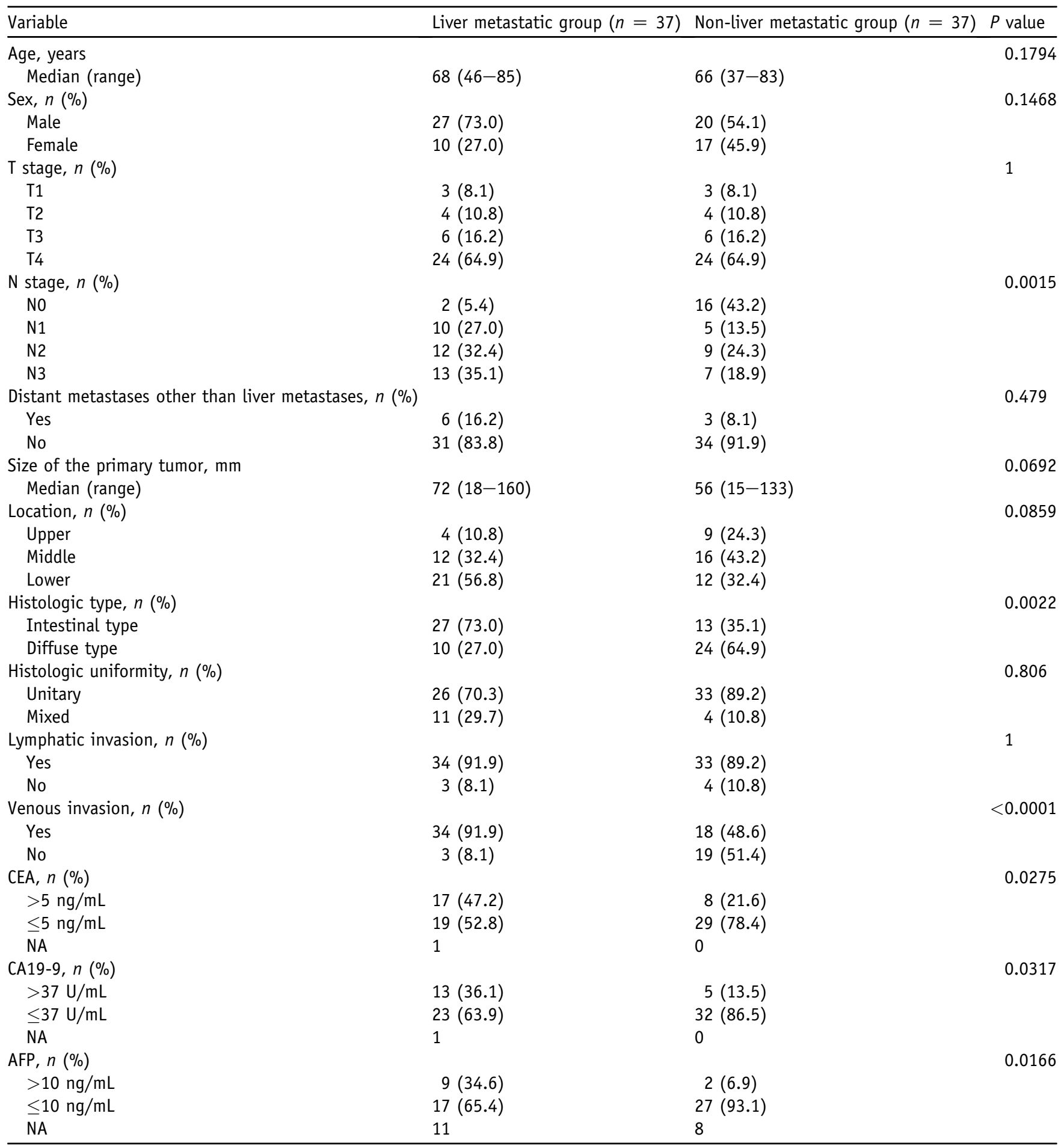

AFP, alpha fetoprotein; CA19-9, carbohydrate antigen 19-9; CEA, carcinoembryonic antigen; LM, liver metastasis; NA, not available.

in the corresponding primary tumors (Figure $1 \mathrm{~A}$ and Supplemental Table S3). Four patients from the metastatic group harbored compound somatic TP53 mutations in their metastatic liver tumors, whereas no patients from the nonmetastatic group had any compound mutation, as described later (Supplemental Table
S3). Thirty-six TP53 mutations were found in tumors of the 32 patients from the metastatic group and included 13 truncating mutations (six nonsense mutations, five frameshift mutations, and two splice site mutations) and 23 nontruncating mutations (22 missense mutations and 1 in-frame deletion) (Supplemental Table S3 and 
Table 3 Somatic Mutations Identified in the Discovery Set

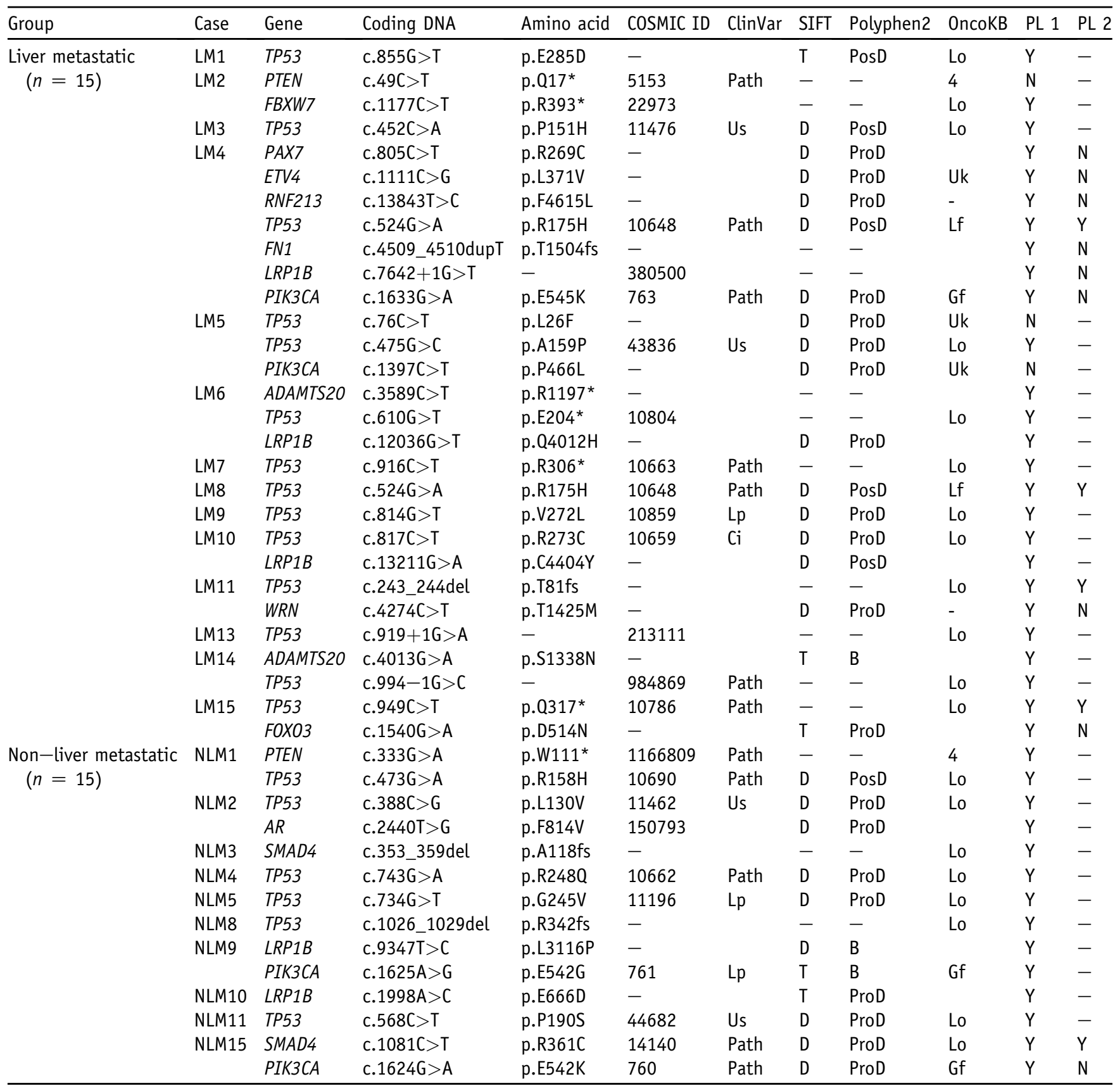

PL1 and PL2 indicate distinctive components separately recognizable in primary tumors. In the columns of PL1 and PL2, Y indicates the presence of mutations in separately recognizable components in primary tumors; $\mathrm{N}$ indicates no mutation in the corresponding component; and - indicates no component separately recognizable.

*Indicates a stop codon.

B, benign; Ci, conflicting interpretation of pathogenicity; D, damaging; Gf, gain-of-function; Lf, loss of function; Lo, likely oncogenic; Lp, likely pathogenic; Path, pathogenic; PosD, possibly damaging; ProD, probably damaging; T, tolerated; Uk, unknown; and Us, uncertain significance.

Supplemental Figure S3). On the other hand, 15 TP53 mutations found in the nonmetastatic group included six truncating mutations (three nonsense mutations, two frameshift mutations, and one splice site mutation) and nine nontruncating mutations (eight missense mutations and one in-frame deletion). The truncating mutations found in the metastatic group were dispersed in the coding regions, whereas most of the nontruncating mutations were in the DNA-binding domain (Figure 1B). Five TP53 mutations (p.L26F, p.H179Y, p.E285D, p.Q354*, and p.H365Y) were found in metastatic tumors, but not in the corresponding primary tumors. Of the 10 patients with heterogeneous primary tumors, mixed histologic features, and metastatic liver 


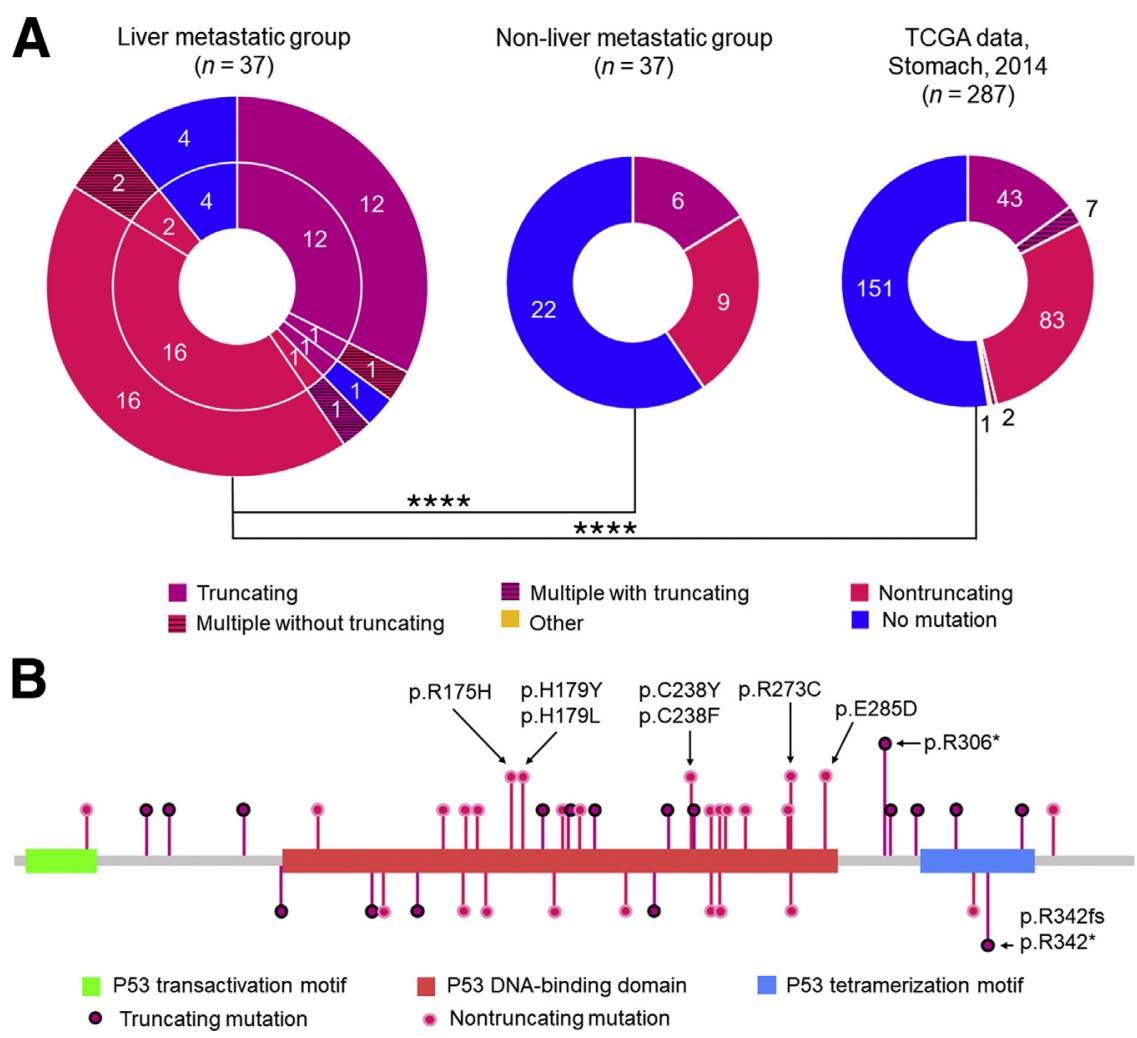

Figure 1 TP53 mutations detected in tumors from 74 patients. A: Genotypes of TP53 in the tumors from the examined patients in the liver metastatic group and the non-liver metastatic group as well as TCGA data for comparison. ${ }^{23-25}$ The inner circle and outer circle in the metastatic group indicate primary tumors and metastatic tumors, respectively. B: Positions and types of mutations plotted on the domain structure for p53 detected in metastatic tumors from the metastatic group (upper) and primary tumors from the nonliver metastatic group (lower). Codons with recurrent mutations are indicated with arrows. Asterisk indicates a stop codon. $n=37$ (liver metastatic group, non-liver metastatic group); $n=287$ (TCGA data). ${ }^{* * * *} P<0.0001$ by Fisher's exact test. tumors with TP53 mutations, 9 shared TP53 mutations among different histologic components of the primary tumor (Supplemental Table S3).

\section{Functional Assessments of TP53 Mutations}

\section{Association between TP53 Mutations and p53 Protein Expression}

All the examined tumors were further investigated for association between TP53 genotype and p53 protein expression by immunohistochemistry. Patterns of p53 expression varied among patients and were classified into overexpression, complete absence of expression or weak expression, cytoplasmic expression, or scattered expression (Figure 2). Truncating mutations in TP53, nontruncating mutations in TP53, and wild-type TP53 seemed to be specifically associated with the complete absence of expression or weak expression, overexpression, and scattered expression of p53, respectively (Table 4). However, some of the mutations in TP53 were associated with alternative patterns of p53 expression (Table 4 and Supplemental Table S3). In truncating mutations, p.N239fs, p.R306*, p.Q317*, and p.R342fs were associated with cytoplasmic expression, suggestive of the impairment of the nuclear import that would lead to the loss of p53 function in nuclei. ${ }^{26}$ c.994$1 \mathrm{G}>\mathrm{C}$ (splice site) and p.R342* were associated with overexpression, indicative of the existence of in-frame products and resistance to nonsense-mediated messenger RNA decay, respectively. However, nontruncating mutations p.H179Y and p.H365Y were associated with complete absence of expression or weak expression, and p.E285D and p.G245V were associated with scattered expression of $\mathrm{p} 53$, suggesting that these missense mutant products are as sensitive to mouse double minute 2 homolog (MDM2) degradation as the wild-type p53.

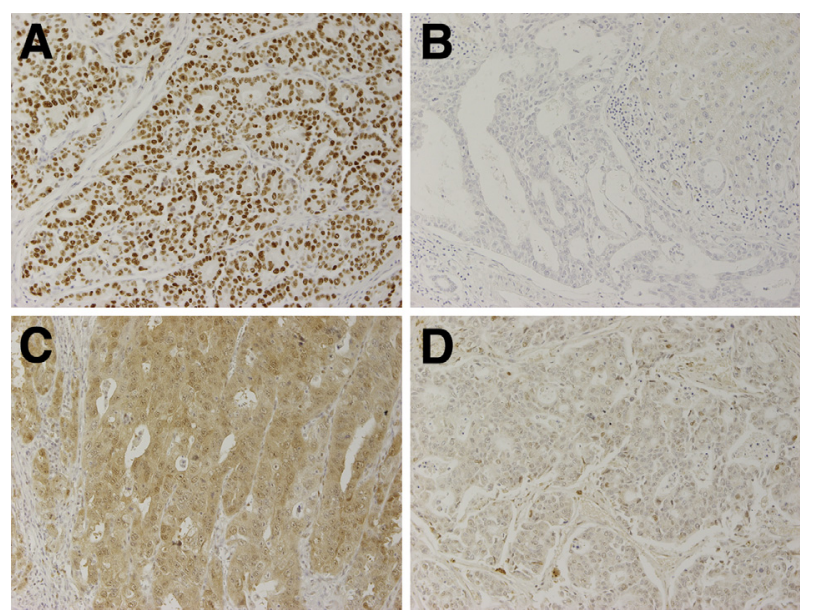

Figure 2 The four patterns of p53 expression detected by immunohistochemistry. A: Overexpression shows diffused, but strong, staining in tumor cell nuclei (as with TP53 ${ }^{\mathrm{R} 175 \mathrm{H}}$ ). B: Complete absence of expression or weak expression shows little to no staining in tumor cell nuclei (as with TP53 ${ }^{\mathrm{E} 204 *}$ ). C: Cytoplasmic expression shows diffused cytoplasmic staining (as with TP53 ${ }^{\mathrm{Q} 317 *}$ ). D: Scattered expression of $\mathrm{p} 53$ shows varying staining intensities in tumor cell nuclei (as with wild-type TP53). Original magnification, $\times 200$. 


\begin{tabular}{|c|c|c|c|c|c|}
\hline \multirow[b]{2}{*}{ Mutation types } & \multicolumn{4}{|c|}{ TP53 expression } & \multirow[b]{2}{*}{ Tota } \\
\hline & Overexpression & Absence/weak & Cytoplasmic & Scattered & \\
\hline \multicolumn{6}{|c|}{ Metastatic liver tumors in the liver metastatic group $(n=37)$} \\
\hline Truncating & 1 & 6 & 5 & 0 & 12 \\
\hline Nontruncating & 17 & 1 & 0 & 1 & 19 \\
\hline Both types & 1 & 0 & 0 & 0 & 1 \\
\hline No mutation & 0 & 0 & 0 & 5 & 5 \\
\hline Total & 19 & 7 & 5 & 6 & 37 \\
\hline \multicolumn{6}{|c|}{ Primary tumors in the liver metastatic group $(n=37)$} \\
\hline Truncating & 1 & 6 & 5 & 2 & 14 \\
\hline Nontruncating & 18 & 0 & 0 & 1 & 19 \\
\hline No mutation & 0 & 0 & 0 & 4 & 4 \\
\hline Total & 19 & 6 & 5 & 7 & 37 \\
\hline \multicolumn{6}{|c|}{ Primary tumors in the non-liver metastatic group $(n=37)$} \\
\hline Truncating & 1 & 2 & 1 & 2 & 6 \\
\hline Nontruncating & 7 & 0 & 0 & 2 & 9 \\
\hline No mutation & 4 & 0 & 0 & 18 & 22 \\
\hline Total & 12 & 2 & 1 & 22 & 37 \\
\hline
\end{tabular}

\section{Alterations in p53 Transcriptional Regulation over Downstream Genes}

The IARC TP53 database ${ }^{21}$ was used for functional assessments of TP53 missense mutations reported in the study cohort, including the 22 mutations found in tumors from 19 patients in the metastatic group and eight mutations found in tumors from eight patients from the nonmetastatic group. Based on the experimental data by Kato et $a{ }^{27},{ }^{27}$ was confirmed that the missense mutations in the tumors of the metastatic group in the study cohort were functionally defective in the protranscriptional activities of p53 on the downstream genes, including $p 21^{W A F 1}(17.9 \%$ activity of wild-type p53; the same hereafter for numbers in paren-

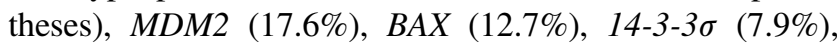
p53AIP1 (7.2\%), GADD45 (4.5\%), Noxa (8.2\%), and p53R2 (14.3\%) (Figure 3). Moreover, some of compound missense mutations in tumors of the study cohort were found to be combinations of a dismissible mutation and a severely impaired mutation, such as p.L26F and p.A159P, p.E285D and p.Y163C, and p.H365Y and p.H179Y. On the other hand, the compound mutations of p.R248Q and p.Q354* were a combination of severely impaired mutations. Missense mutations found in eight tumors from the nonmetastatic group were also found to be functionally defective, yet the frequency of missense mutations in the nonmetastatic group was much lower than that in the metastatic group $(P=0.0150)$.

\section{Correlations between TP53 Mutations and Clinicopathologic Features}

TP53 mutations found in the metastatic group were positively associated with $\mathrm{N}$ stage, dominant histologic grade of the primary tumor, venous invasion, and serum levels of AFP and CEA. In addition, TP53 mutations in the metastatic group were negatively associated with the histologic uniformity of the primary tumor (Table 5).

Subgroup Analysis Discriminating between the Presence and Absence of Distant Metastasis Other than Liver Metastasis

Herein, the molecular alterations specifically associated with liver metastasis from gastric cancer irrespective of any other distant metastases were investigated. Distant metastases other than liver metastases were observed in six of 37 patients with liver metastasis and three of 37 patients without liver metastasis (Supplemental Table S2 and Table 2). A subgroup analysis was conducted by dividing the patients into four cohorts depending on their metastatic states, namely, patients without any distant metastases $(n=34)$, those with liver metastases but no other distant metastases $(n=31)$, those with liver and other distant metastases $(n=6)$, and those with distant metastases other than liver metastases $(n=3)$ (Supplemental Table S4). TP53 mutations were significantly more frequent in the patients with liver metastases but no other distant metastases $(90.3 \%$; $28 /$ $31)$ than in those without any distant metastases $(44.1 \% ; 15 /$ $34 ; P<0.0001)$ or those with distant metastases other than liver metastases $(0 \% ; 0 / 3 ; P=0.0033)$.

\section{Discussion}

The investigation of multiple cancer-associated genes revealed the frequent somatic mutations in TP53 as well as miscellaneous somatic mutations in LRPIB, PIK3CA, ADAMTS20, PAX7, FN1, FOXO3, WRN, PTEN, ETV4, and $R N F 213$ in metastatic liver tumors from gastric cancer.

The gene TP53 was frequently mutated, as $86.5 \%$ of the patients from the metastatic group harbored TP53 


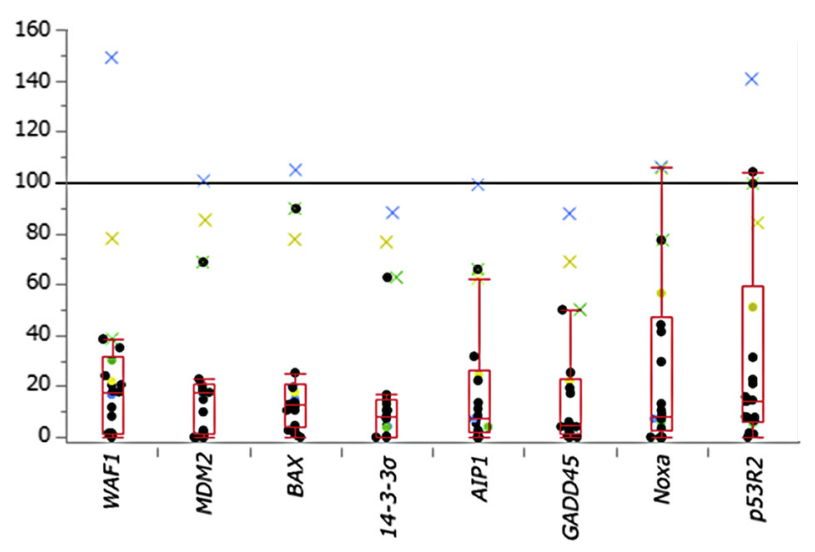

Figure 3 Functional assessment of TP53 missense mutations detected in the metastatic liver tumors collated with data from the IARC TP53 Database. ${ }^{21}$ The vertical axis represents the percentage of promoter-specific transcriptional activity in missense mutations for each downstream gene relative to wild-type activity. Box and whisker plots represent the median, upper, and lower quartiles, and maximum and minimum values excluding outliers. Black dots indicate missense mutations found in liver metastases. Colored crosses and dots indicate compound mutations found in liver metastases, namely, p.L26F (blue cross) and p.A159P (blue dot), p.E285D (green cross) and p.Y163C (green dot), and p.H365Y (yellow cross) and p.H179Y (yellow dot).

mutations in their metastatic liver tumors. The frequency of metastatic TP53 mutations in the metastatic group was more than twice that in the nonmetastatic group (40.5\%) and even far more common than the average frequency in gastric cancers, as evidenced from the comparison with TCGA data (47.4\%). These results indicate that the mutant p53 is an important prometastatic molecule for the metastasis of gastric cancer to the liver. Most TP53 mutations in the metastatic tumors were shared by corresponding primary tumors, but some mutations were only found in the metastatic tumors, suggestive of the role of TP53 mutations in gastric cancer in the development and growth of metastases. Moreover, most TP53 mutations in metastatic tumors from heterogeneous primary tumors with mixed histologic features were shared among histologically different components. Thus, the liver-tropic

Table 5 Spearman's Correlation between TP53 Mutations Identified in Liver Metastasis and Clinicopathologic Features

\begin{tabular}{lrr}
\hline Variable & Rho & $P$ value \\
\hline Node metastasis & 0.33 & 0.0037 \\
Distant metastasis other than liver & 0.22 & 0.0575 \\
Size of primary tumors & 0.21 & 0.0712 \\
Dominant histologic grade of primary tumors & 0.25 & 0.0307 \\
Histologic uniformity of primary tumors & -0.28 & 0.0168 \\
Lymphatic invasion & 0.02 & 0.8655 \\
Venous invasion & 0.42 & 0.0002 \\
Serum level of CEA at gastrectomy & 0.33 & 0.0043 \\
Serum level of CA19-9 at gastrectomy & 0.18 & 0.1216 \\
Serum level of AFP at gastrectomy & 0.37 & 0.0061 \\
\hline
\end{tabular}

AFP, alpha fetoprotein; CA19-9, carbohydrate antigen 19-9; CEA, carcinoembryonic antigen. property may be determined early during primary gastric cancer development by positive selection before the subsequent heterogeneous clonal evolution of the tumor. This observation may be consistent with a previous report indicating that TP53 mutations could be observed in the inflamed gastric mucosa with Helicobacter pylori infection even before the development of carcinoma. ${ }^{28}$ On the other hand, more than half of the gastric cancers without liver metastasis had no TP53 mutation, suggesting that TP53 mutations may not be essential for gastric cancer development but rather endow additional properties necessary for the prometastatic ability of primary tumors.

Among the miscellaneous mutations, LRPIB, PIK3CA, and ADAMTS 20 were recurrently mutated in the metastatic liver tumors. $L R P 1 B$, encoding a member of the low-density lipoprotein receptor family, has been reported to suppress growth, colony formation, and tumor formation of gastric cancer cells xenografted into nude mice. ${ }^{29}$ LRPIB mutations observed in the study cohort included a splice site mutation and missense mutations at diverse positions. PIK3CA, encoding the $\mathrm{p} 110 \alpha$ subunit of phosphatidylinositol-4,5bisphosphate 3-kinase, carries mutational hotspots (E542K, E545K, and H1047R) with a gain of enzymatic function inducing protein kinase B (AKT) activation. ${ }^{30}$ These hotspot mutations were observed in the two groups of the study cohort. ADAMTS20, encoding a member of the ADAMTS family of zinc-dependent proteases, is thought to be essential for murine melanoblast migration. ${ }^{31}$ In the study cohort, however, one of the two mutations was a nonsense mutation, indicating that the loss-of-function mutation in ADAMTS20 may also play a role in the course of metastasis. Hence, further studies are warranted to clarify the precise cancer-associated functions of this gene. Consistent with the results of other studies that showed $L R P I B$ and PIK3CA as commonly mutated genes in the primary gastric cancer, ${ }^{23-25}$ recurrent mutations were also observed in these genes among the patients from the nonmetastatic group. However, as LRPIB, PIK3CA, and ADAMTS20 always coexisted with TP53 mutations in the metastatic group, but not in the nonmetastatic group, these mutations may promote liver metastasis in conjunction with the mutant TP53.

LRPIB, PIK3CA, PAX7, FN1, FOXO3, WRN, ETV4, and $R N F 213$ were mutated in metastatic liver tumors but also appeared to be positively selected during metastasis from a heterogeneous primary tumor showing mixed histologic features (cells with and without the mutation). This finding suggests that these clonally selected gene mutations may confer advantages for the development of liver metastasis.

Whole-genome sequencing examination showed that primary gastric cancers may be classified into four molecular subtypes, namely, Epstein-Barr virus-positive, microsatellite instability, genomically stable, and chromosomal instability. ${ }^{23}$ Among these subtypes, TP53 mutations were accumulated in $71 \%$ of gastric carcinomas of the chromosomal instability type. Although this whole-genome study 
revealed no data regarding liver metastases, gastric cancers of the chromosomal instability type might cause liver metastasis. The whole-genome study also indicated that gastric cancers of the chromosomal instability type showed complex copy number variations; however, the analysis failed to provide reliable copy number variation data, owing to the fragmented DNA obtained from FFPE tissues.

To associate molecular basis with potential therapeutic targets that are versatile in liver metastasis of gastric cancer, it is important to specify, not only the responsible genes, but also the aberrant pathways that are commonly involved in metastatic tumors. By integrating the experimental data and functional assessments of mutated TP53 in the IARC TP53 Database, some specific downstream pathways of p53 were confirmed. First, truncating mutations of TP53 were detected in 40.6\% (13/32) of the patients that harbored TP53 mutations in their metastatic liver tumors; thus, the mutations were more frequent than the $19.6 \%(5668 / 28,869)$ frequency of TP53 truncating mutations found in the database. $^{21}$ Truncating mutations of TP53 in the study cohort associated with complete absence of expression or weak expression of p53 protein may result in its loss of function. In addition, the complete absence of expression or weak expression of p53 was more common in the study cohort, as observed in seven metastatic liver tumors from the metastatic patients, six primary tumors from the metastatic patients, and two primary tumors from the nonmetastatic patients. These results suggest that the loss of p53 function may provide a great advantage for the development and/or growth of liver metastasis from gastric cancer. In addition, the ratio of patients harboring nontruncating mutations to truncating ones was not higher in the metastatic group than in the nonmetastatic group. This observation would corroborate the contribution of loss of p53 function to liver metastasis from gastric cancer because the positive contribution of TP53 gain-of-function to liver metastasis would have led to a higher ratio in the metastatic group than in the nonmetastatic group. Second, the missense mutations of TP53 found in the metastatic tumors produced functionally defective proteins, as evidenced by Kato et $\mathrm{al}^{27}$ who demonstrated deficient protranscriptional activities of $\mathrm{p} 53$ mutants with respect to $p 21^{W A F 1}, M D M 2, B A X, 14-3-3 \sigma$, p53AIP1, GADD45, Noxa, and p53R2; the corresponding downstream pathways would cause i) cell cycle arrest, ${ }^{32-34}$ ii) apoptosis, ${ }^{35-37}$ iii) DNA repair and damage prevention, ${ }^{38,39}$ and iv) cellular senescence ${ }^{40}$ (Supplemental Figure S4). Most of the TP53 missense mutations were associated with the overexpression of p53 in the metastatic tumors in the study cohort, thereby proving that these mutated p53 proteins had accumulated in the nucleus and functioned in the dominant-negative manner. ${ }^{41,42}$ These results indicate that the consistent dysregulated nuclear pathways downstream of $\mathrm{p} 53$ play a major role during liver metastasis from gastric cancer.

The assessment of the relationship between TP53 mutations and clinicopathologic features revealed the association between TP53 mutations in the metastatic group and poor prognostic factors previously reported, including venous invasion, nodal state, elevated serum AFP level, and mixed histology. ${ }^{12,43,44}$ Of these, increased venous invasion, a process important to facilitate hematogenous metastasis such as liver metastasis, could reflect the deregulated inhibition of angiogenesis owing to TP53 mutation. ${ }^{45}$ These multiple correlated factors suggest that the TP53 mutations found in the metastatic group have multiple negative effects on clinical prognosis of gastric cancer, which could be simultaneously improved upon correction or treatment of TP53-related pathways associated with liver metastasis of gastric cancer. Such options may soon be practical, as therapeutic approaches to repair the defective p53 pathway are now in the process of development. ${ }^{46-50}$

In summary, among the 412 cancer-associated genes, TP53 mutations were most frequently involved in liver metastasis from gastric cancer. TP53 mutations were assessed as loss-of-function mutations rather than gain-offunction ones. Moreover, TP53 mutations in the metastatic tumors were well shared by primary tumors, indicating a diagnostic window for mutations in distant metastases from primary tumors that would be easily accessible. These results suggest that the p53 pathway should be the prime focus for the development of biomarkers and therapeutic approaches to treat the liver metastasis of gastric cancer.

\section{Acknowledgments}

We thank Yoji Nagashima, Takao Ito, Yuzuri Hasegawa, Aya Shiraishi, Kaori Murakami, and Hiroshi Ochiai for preparing FFPE sections, and Yuji Suehiro, Tomoko Motohashi, Sawako Yoshina, Etsuko Tanji, and Hiroyuki Akagawa for technical assistance with next-generation sequencing and data analysis.

N.I., A.S., and T.F. conceived the study and designed the experiments; M.Y. coordinated sample acquisition; N.I., A.S., and M.Y. acquired clinical data; N.I. performed experiments; N.I., A.S., S.M., and T.F. performed the bioinformatics data analysis; N.I. and A.S. wrote the manuscript; S.M., M.Y., and T.F. reviewed the manuscript; all authors approved the final manuscript.

\section{Supplemental Data}

Supplemental material for this article can be found at https://doi.org/10.1016/j.ajpath.2018.12.015.

\section{References}

1. Tanabe S, Ishido K, Matsumoto T, Kosaka T, Oda I, Suzuki H, Fujisaki J, Ono H, Kawata N, Oyama T, Takahashi A, Doyama H, Kobayashi M, Uedo N, Hamada K, Toyonaga T, Kawara F, Tanaka S, Yoshifuku Y: Long-term outcomes of endoscopic 
submucosal dissection for early gastric cancer: a multicenter collaborative study. Gastric Cancer 2017, 20 Suppl 1:45-52

2. Yoshimura F, Inaba K, Kawamura Y, Ishida Y, Taniguchi K, Isogaki J, Satoh S, Kanaya S, Sakurai Y, Uyama I: Clinical outcome and clinicopathological characteristics of recurrence after laparoscopic gastrectomy for advanced gastric cancer. Digestion 2011, 83:184-190

3. Koizumi W, Narahara H, Hara T, Takagane A, Akiya T, Takagi M, Miyashita K, Nishizaki T, Kobayashi O, Takiyama W, Toh Y, Nagaie T, Takagi S, Yamamura Y, Yanaoka K, Orita H, Takeuchi M: S-1 plus cisplatin versus S-1 alone for first-line treatment of advanced gastric cancer (SPIRITS trial): a phase III trial. Lancet Oncol 2008, 9:215-221

4. Maehara Y, Hasuda S, Koga T, Tokunaga E, Kakeji Y, Sugimachi K: Postoperative outcome and sites of recurrence in patients following curative resection of gastric cancer. Br J Surg 2000, 87:353-357

5. Honda M, Hiki N, Kinoshita T, Yabusaki H, Abe T, Nunobe S, Terada M, Matsuki A, Sunagawa H, Aizawa M, Healy MA, Iwasaki M, Furukawa TA: Long-term outcomes of laparoscopic versus open surgery for clinical stage I gastric cancer: the LOC-1 study. Ann Surg 2016, 264:214-222

6. Zhang W, Yu Y, Fang Y, Wang Y, Cui Y, Shen K, Liu T: Systemic chemotherapy as a main strategy for liver metastases from gastric cancer. Clin Transl Oncol 2015, 17:888-894

7. Japanese Gastric Cancer Association: Japanese gastric cancer treatment guidelines 2014 (ver. 4). Gastric Cancer 2017, 20:1-19

8. De Manzoni G, Marrelli D, Baiocchi GL, Morgagni P, Saragoni L, Degiuli M, Donini A, Fumagalli U, Mazzei MA, Pacelli F, Tomezzoli A, Berselli M, Catalano F, Di Leo A, Framarini M, Giacopuzzi S, Graziosi L, Marchet A, Marini M, Milandri C, Mura G, Orsenigo E, Quagliuolo V, Rausei S, Ricci R, Rosa F, Roviello G, Sansonetti A, Sgroi G, Tiberio GA, Verlato G, Vindigni C, Rosati R, Roviello F: The Italian Research Group for Gastric Cancer (GIRCG) guidelines for gastric cancer staging and treatment: 2015. Gastric Cancer 2017, 20:20-30

9. Kodera Y, Fujitani K, Fukushima N, Ito S, Muro K, Ohashi N, Yoshikawa T, Kobayashi D, Tanaka C, Fujiwara M: Surgical resection of hepatic metastasis from gastric cancer: a review and new recommendation in the Japanese gastric cancer treatment guidelines. Gastric Cancer 2014, 17:206-212

10. Zhang J, Huang JY, Chen YN, Yuan F, Zhang H, Yan FH, Wang MJ, Wang G, Su M, Lu G, Huang Y, Dai H, Ji J, Zhang J, Zhang JN, Jiang YN, Chen SJ, Zhu ZG, Yu YY: Whole genome and transcriptome sequencing of matched primary and peritoneal metastatic gastric carcinoma. Sci Rep 2015, 5:13750

11. Liu H, Li F, Zhu Y, Li T, Huang H, Lin T, Hu Y, Qi X, Yu J, Li G: Whole-exome sequencing to identify somatic mutations in peritoneal metastatic gastric adenocarcinoma: a preliminary study. Oncotarget 2016, 7:43894-43906

12. Ikari N, Taniguchi $K$, Serizawa A, Yamada T, Yamamoto $M$, Furukawa T: A primary tumor of mixed histological type is a novel poor prognostic factor for patients undergoing resection of liver metastasis from gastric cancer. J Hepatobiliary Pancreat Sci 2017, 24 281-288

13. Ajani JA, In H, Sano $T$, Gaspar LE, Erasmus JJ, Tang LH, Washington MK, Gerdes H, Wittekind CW, Mansfield PF, Rimmer C, Hofstetter WL, Kelsen D: Stomach. Edited by Amin MB, Edge S, Greene F, Byrd DR, Brookland RK, Washington MK, Gershenwald JE, Compton CC, Hess KR, Sullivan DC, Jessup JM, Brierley JD, Gaspar LE, Schilsky RL, Balch CM, Winchester DP, Asare EA, Madera M, Gress DM, Meyer LR. In AJCC Cancer Staging Manual. ed 8. New York, NY: Springer, 2017. pp. 203-220

14. Ikari N, Aoyama S, Seshimo A, Suehiro Y, Motohashi T, Mitani S, Yoshina S, Tanji E, Serizawa A, Yamada T, Taniguchi K, Yamamoto M, Furukawa T: Somatic mutations and increased lymphangiogenesis observed in a rare case of intramucosal gastric carcinoma with lymph node metastasis. Oncotarget 2018, 9:10808-10817

15. D'Haene N, Le Mercier M, De Neve N, Blanchard O, Delaunoy M, El Housni H, Dessars B, Heimann P, Remmelink M, Demetter P,
Tejpar S, Salmon I: Clinical validation of targeted next generation sequencing for colon and lung cancers. PLoS One 2015, 10:e0138245

16. Landrum MJ, Lee JM, Benson M, Brown G, Chao C, Chitipiralla S, Gu B, Hart J, Hoffman D, Hoover J, Jang W, Katz K, Ovetsky M, Riley G, Sethi A, Tully R, Villamarin-Salomon R, Rubinstein W, Maglott DR: ClinVar: public archive of interpretations of clinically relevant variants. Nucleic Acids Res 2016, 44:D862-D868

17. Kumar P, Henikoff S, Ng PC: Predicting the effects of coding nonsynonymous variants on protein function using the SIFT algorithm. Nat Protoc 2009, 4:1073-1081

18. Adzhubei I, Jordan DM, Sunyaev SR: Predicting functional effect of human missense mutations using PolyPhen-2. Curr Protoc Hum Genet 2013, 76:7.20.1-7.20.41

19. Chakravarty D, Gao J, Phillips SM, Kundra R, Zhang H, Wang J, et al: OncoKB: a precision oncology knowledge base. JCO Precis Oncol 2017, 2017. 10.1200/PO.17.00011

20. Kuboki Y, Shimizu K, Hatori T, Yamamoto M, Shibata N, Shiratori K, Furukawa T: Molecular biomarkers for progression of intraductal papillary mucinous neoplasm of the pancreas. Pancreas 2015, 44:227-235

21. Bouaoun L, Sonkin D, Ardin M, Hollstein M, Byrnes G, Zavadil J, Olivier M: TP53 variations in human cancers: new lessons from the IARC TP53 database and genomics data. Hum Mutat 2016, 37: 865-876

22. Lauren P: The two histological main types of gastric carcinoma: diffuse and so-called intestinal-type carcinoma. An attempt at a histoclinical classification. Acta Pathol Microbiol Scand 1965, 64:31-49

23. Cancer Genome Atlas Research Network: Comprehensive molecular characterization of gastric adenocarcinoma. Nature 2014, 513: 202-209

24. Gao J, Aksoy BA, Dogrusoz U, Dresdner G, Gross B, Sumer SO, Sun Y, Jacobsen A, Sinha R, Larsson E, Cerami E, Sander C, Schultz N: Integrative analysis of complex cancer genomics and clinical profiles using the cBioPortal. Sci Signal 2013, 6:p11

25. Cerami E, Gao J, Dogrusoz U, Gross BE, Sumer SO, Aksoy BA, Jacobsen A, Byrne CJ, Heuer ML, Larsson E, Antipin Y, Reva B, Goldberg AP, Sander C, Schultz N: The cBio cancer genomics portal: an open platform for exploring multidimensional cancer genomics data. Cancer Discov 2012, 2:401-404

26. Liang SH, Clarke MF: The nuclear import of p53 is determined by the presence of a basic domain and its relative position to the nuclear localization signal. Oncogene 1999, 18:2163-2166

27. Kato S, Han SY, Liu W, Otsuka K, Shibata H, Kanamaru R, Ishioka C: Understanding the function-structure and functionmutation relationships of p53 tumor suppressor protein by highresolution missense mutation analysis. Proc Natl Acad Sci U S A 2003, 100:8424-8429

28. Shimizu T, Marusawa H, Matsumoto Y, Inuzuka T, Ikeda A, Fujii Y, Minamiguchi S, Miyamoto S, Kou T, Sakai Y, Crabtree JE, Chiba T: Accumulation of somatic mutations in TP53 in gastric epithelium with Helicobacter pylori infection. Gastroenterology 2014, 147: 407-417.e403

29. Lu YJ, Wu CS, Li HP, Liu HP, Lu CY, Leu YW, Wang CS, Chen LC, Lin KH, Chang YS: Aberrant methylation impairs low density lipoprotein receptor-related protein $1 \mathrm{~B}$ tumor suppressor function in gastric cancer. Genes Chromosomes Cancer 2010, 49:412-424

30. Bader AG, Kang S, Vogt PK: Cancer-specific mutations in PIK3CA are oncogenic in vivo. Proc Natl Acad Sci U S A 2006, 103:1475-1479

31. Silver DL, Hou L, Somerville R, Young ME, Apte SS, Pavan WJ: The secreted metalloprotease ADAMTS20 is required for melanoblast survival. PLoS Genet 2008, 4:e1000003

32. el-Deiry WS, Tokino T, Velculescu VE, Levy DB, Parsons R, Trent JM, Lin D, Mercer WE, Kinzler KW, Vogelstein B: WAF1, a potential mediator of p53 tumor suppression. Cell 1993, 75:817-825

33. Chan TA, Hermeking H, Lengauer C, Kinzler KW, Vogelstein B: 14 3-3Sigma is required to prevent mitotic catastrophe after DNA damage. Nature 1999, 401:616-620 
34. Jin S, Tong T, Fan W, Fan F, Antinore MJ, Zhu X, Mazzacurati L, Li X, Petrik KL, Rajasekaran B, Wu M, Zhan Q: GADD45-induced cell cycle G2-M arrest associates with altered subcellular distribution of cyclin B1 and is independent of p38 kinase activity. Oncogene 2002, 21:8696-8704

35. Miyashita T, Reed JC: Tumor suppressor p53 is a direct transcriptional activator of the human bax gene. Cell 1995, 80:293-299

36. Oda E, Ohki R, Murasawa H, Nemoto J, Shibue T, Yamashita T, Tokino T, Taniguchi T, Tanaka N: Noxa, a BH3-only member of the Bcl-2 family and candidate mediator of p53-induced apoptosis. Science 2000, 288:1053-1058

37. Oda K, Arakawa H, Tanaka T, Matsuda K, Tanikawa C, Mori T, Nishimori H, Tamai K, Tokino T, Nakamura Y, Taya Y: p53AIP1, a potential mediator of p53-dependent apoptosis, and its regulation by Ser-46-phosphorylated p53. Cell 2000, 102:849-862

38. Hollander MC, Kovalsky O, Salvador JM, Kim KE, Patterson AD, Haines DC, Fornace AJ Jr: Dimethylbenzanthracene carcinogenesis in Gadd45a-null mice is associated with decreased DNA repair and increased mutation frequency. Cancer Res 2001, 61:2487-2491

39. Tanaka H, Arakawa H, Yamaguchi T, Shiraishi K, Fukuda S, Matsui K, Takei Y, Nakamura Y: A ribonucleotide reductase gene involved in a p53-dependent cell-cycle checkpoint for DNA damage. Nature 2000, 404:42-49

40. Hofseth LJ, Hussain SP, Harris CC: p53: 25 years after its discovery. Trends Pharmacol Sci 2004, 25:177-181

41. Lukashchuk N, Vousden KH: Ubiquitination and degradation of mutant p53. Mol Cell Biol 2007, 27:8284-8295

42. Kamada R, Toguchi $Y$, Nomura $T$, Imagawa $T$, Sakaguchi K: Tetramer formation of tumor suppressor protein p53: structure, function, and applications. Biopolymers 2016, 106:598-612
43. Reim D, Choi YS, Yoon HM, Park B, Eom BW, Kook MC, Ryu KW, Choi IJ, Joo J, Kim YW: Alpha-fetoprotein is a significant prognostic factor for gastric cancer: results from a propensity score matching analysis after curative resection. Eur J Surg Oncol 2017, 43:1542-1549

44. Ohno S, Fujii T, Ueda S, Nakamoto T, Kinugasa S, Yoshimura H, Tachibana M, Kubota H, Kumar Dhar D, Nagasue N: Predictive factors and timing for liver recurrence after curative resection of gastric carcinoma. Am J Surg 2003, 185:258-263

45. Tokino T, Nakamura Y: The role of p53-target genes in human cancer. Crit Rev Oncol Hematol 2000, 33:1-6

46. Lehmann S, Bykov VJ, Ali D, Andren O, Cherif H, Tidefelt U, Uggla B, Yachnin J, Juliusson G, Moshfegh A, Paul C, Wiman KG, Andersson PO: Targeting p53 in vivo: a first-in-human study with p53-targeting compound APR-246 in refractory hematologic malignancies and prostate cancer. J Clin Oncol 2012, 30:3633-3639

47. Martin L, Grigoryan A, Wang D, Wang J, Breda L, Rivella S, Cardozo T, Gardner LB: Identification and characterization of small molecules that inhibit nonsense-mediated RNA decay and suppress nonsense p53 mutations. Cancer Res 2014, 74:3104-3113

48. Khoo KH, Verma CS, Lane DP: Drugging the p53 pathway: understanding the route to clinical efficacy. Nat Rev Drug Discov 2014, 13: $217-236$

49. Kamino H, Nakamura Y, Tsuneki M, Sano H, Miyamoto Y, Kitamura N, Futamura M, Kanai Y, Taniguchi H, Shida D, Kanemitsu Y, Moriya Y, Yoshida K, Arakawa H: Mieap-regulated mitochondrial quality control is frequently inactivated in human colorectal cancer. Oncogenesis 2016, 4:e181

50. Morton DJ, Patel D, Joshi J, Hunt A, Knowell AE, Chaudhary J: ID4 regulates transcriptional activity of wild type and mutant p53 via K373 acetylation. Oncotarget 2017, 8:2536-2549 